\title{
BREADTH TWO TOPOLOGICAL LATTICES WITH CONNECTED SETS OF IRREDUCIBLES
}

\author{
BY
}

J. W. LEA, JR.

ABSTRACT. Breadth two topological lattices with connected sets of irreducible elements are characterized by these sets.

1. Introduction. In the study of topological lattices and semilattices on the two cell, the boundary arcs have played an important role. A. D. Wallace [18] , D. R. Brown [4] , and A. Y. W. Lau [8] have given conditions under which the boundary generates the lattice or semilattice. J. B. Rhodes [16] has shown that two such lattices are iseomorphic (homeomorphic and isomorphic) if their boundaries are order isomorphic. Rhodes, for metric lattices, and the author, in the general case, have found that the meet and join irreducible elements are contained in the boundary [16], [13].

In this article we establish conditions for the boundary of a topological lattice on the two cell to consist entirely of irreducible elements. These lattices may then be characterized by their chains of irreducible elements.

In \$4 we shall extend the study to nondistributive lattices of breadth two.

The only use made of the metric condition is that any two nondegenerate arc chains (compact connected chains) are iseomorphic. Thus the results are valid in any class of lattices having this property.

2. Preliminaries. The width $w(X)$ of a partially ordered set $X$ is the maximum number of elements in a set of incomparable elements. For $x \in X$ we let $M(x)=\{y \in X: x \leqslant y\}$ and $L(x)=\{y \in X: y \leqslant x\} ;$ when $x \leqslant y$, then $[x, y]$ $=M(x) \cap L(y)$.

Let $L$ be a bounded lattice and $C$ a chain in $L$ which contains 1 and which is closed under arbitrary meets in $L$. Then $s_{c}: L \rightarrow C$ is defined by $s_{c}(x)=$ $\bigwedge\{y \in C: x \leqslant y\}$ for all $x \in L$. In [2] it is shown that $s_{c}$ is a join homomorphism, $x \leqslant s_{c}(x)$ for all $x \in L, s_{c}(p)=p$ for all $p \in C$; also $s_{c}$ is a meet homomorphism when $C$ consists of prime elements. We shall use $t_{c}$ for the dual notion.

Received by the editors October 23, 1974 and, in revised form, May 28, 1975. $22 \mathrm{~A} 30$.

AMS (MOS) subject classifications (1970). Primary 06A30, 06A35, 54 F05; Secondary

Key words and phrases. Topological lattice, meet (join) irreducible. 
We let $M(L)[J(L)]$ denote the set of all meet [join] irreducible elements of $L$; also $A^{*}$ and $A^{\circ}$ denote, respectively, the topological closure and interior of $A$.

3. Distributive lattices. The two goals of this section are to establish conditions under which $M(L) \cup J(L)$ is the whole boundary of $L$ and to characterize $L$ by $M(L) \cup J(L)$.

THEOREM 3.1. Let $L$ be a compact topological lattice of finite breadth with $M(L)$ connected. If no meet irreducible is a cutpoint of $L$, then $L$ has no cutpoints.

Proof. If the breadth of $L$ is $n$, then the continuous function $(M(L))^{n}$ $\rightarrow L$ defined by $\left(x_{1}, \ldots, x_{n}\right) \mapsto x_{1} \wedge \cdots \wedge x_{n}$ is onto [2]. Thus $L$ is connected. Suppose $p \in L$ is a cutpoint. Then $L=L(p) \cup M(P)$ [1]. Since $1 \in M(L)$, then $M(L) \cap(M(p) \backslash\{p\}) \neq \varnothing$. Let $x \in L(p) \backslash\{p\}$ and let $x=x_{1} \wedge \cdots \wedge x_{n}$ with all $x_{i} \in M(L)$. For some $i$, we have $x_{i} \in L(p) \backslash\{p\}$; hence $M(L) \cap(L(p) \backslash\{p\}) \neq \varnothing$. Thus $M(L)$ has a nontrivial separation. But $M(L)$ is connected; therefore $L$ has no cutpoints.

THEOREM 3.2. Let $L$ be a compact connected topological lattice of finite breadth with $w(M(L))=2$. Then $L$ is iseomorphic to a sublattice of a direct product of two arc chains.

Proof. The breadth of $L$ is two [12] and so $L$ is modular [6]. Thus $L$ is distributive [3, p. 66]; hence the conclusion follows [2].

A lattice has meet (join) representations if each element is a meet (join) of meet (join) irreducibles.

THEOREM 3.3. Let $L$ be a bounded distributive lattice of breadth two which has finite irredundant meet and join representations and for which $w(M(L))=2$. Then $M(L) \cap J(L) \neq \varnothing$.

Proof. If $0 \in M(L)$, we are done. Suppose $\{x, y\} \subset M(L)$ is meet irredundant and $0=x \wedge y$. Suppose also that $x=a \vee b$ with $a, b \in J(L)$. If $a=$ $a_{1} \wedge a_{2}$ and $b=b_{1} \wedge b_{2}$ with $a_{1}, a_{2}, b_{1}$, and $b_{2} \in M(L)$, then $0=x \wedge y \geqslant$ $a \wedge y=a_{1} \wedge a_{2} \wedge y$; thus $0=a_{1} \wedge y$ or $0=a_{2} \wedge y$ or $0=a_{1} \wedge a_{2}$. If the latter case holds we are done since then $x=b \in J(L)$. Thus suppose $0=a_{1} \wedge y$. Since $L$ is distributive, $x=a_{1}[3, \mathrm{p} .58]$. In a similar manner $x=b_{1}$ or $b_{2}$; say $x=b_{2}$. Since $w(M(L))=2$, two of $a_{1}, a_{2}, b_{1}$ must be comparable. It is now easily verified that $a, b$, or $x$ must belong to $M(L) \cap J(L)$.

Definition 3.4. If $L$ is a lattice with 1 and $C$ is a chain in $L$, then $C$ is a coordinate chain of $L$ if

(i) $1 \in C$, 
(ii) $C$ is closed under arbitrary meets in $L$, and

(iii) $x \in C$ implies $x=\wedge D$ for some $D \subset M(L) \cap C$.

A marginal element of a space is an element of the cohomological bound. ary (sce [10]).

THEOREM 3.5. Let $L$ be a compact connected distributive topological lattice of breadth two. Let $A$ and $B$ be coordinate chains for $L$ and $C$ and $D$ their respective extensions to maximal chains from 0 to 1 . If $p \notin C \cup D$, then $p$ is not marginal in $L$.

Proof. Let $p \in L \backslash(C \cup D)$. Then $p=s_{A}(p) \wedge s_{B}(p)$. Under the iseomorphism mentioned in Theorem 3.2, the image of $p$ in $A \times B$ is $\left(s_{A}(p), s_{B}(p)\right) \in$ $(\operatorname{lm}(L))^{\circ}$. Since $p \notin A \cup B$, then $s_{A}(p)\left(s_{B}(p)\right)$ is not marginal in $A(B)$. Therefore $\left(s_{A}(p), s_{B}(p)\right)$ is not marginal in $A \times B$ [10]. Thus $p$ is not marginal in $L$.

K. Oberhoff has obtained a result similar to Theorem 3.5 assuming that the underlying space of $L$ is the product of two arc chains [15].

TheORFM 3.6. Let $L$ be a compact topological lattice of finite breadth with no meet irreducible cutpoints. If $M(L)$ and $J(L)$ are both the union of two arc chains, then the set $B$ of all marginal elements of $L$ is equal to $M(L) \cup J(L)$.

Proor. That $M(L) \cup J(L) \subset B$ follows from Theorem 3.1 above and Theorem 2.1 of [13]. Let $C$ and $D$ be coordinate chains and extend $C$ and $D$ to maximal chains $S$ and $T$ from 0 to 1. Let $x \in B \backslash\{0,1\}$. By Theorem 3.5, $x \in$ $S \cup T$. Suppose $x \in S \backslash(M(L) \cup J(L))$. By Theorem 3.3, there exists an element $y \in M(L) \cap J(L) \cap S$. Either $x<y$ or $y<x$. If $x<y$, let $x=s \vee t$ with $s \in$ $J(L) \cap S$ and $t \in J(L) \cap T$. Then $[J(L) \cap S \cap L(X)] \cup[J(L) \cap S \cap M(x)]$ is a nontrivial separation of the connected set $J(L) \cap S$. Thus $B \subset M(L) \cup J(L)$.

If $S$ and $T$ are the chains in Theorem 3.6, then it is easily verified that $L=$ $S \wedge T=S \vee T$ and $S \cap T=\{0,1\}$.

We shall now characterize the lattices of Theorem 3.6. If $A=M \cup J$ is an arc chain from 0 to 1 in a lattice $L$ with $M$ and $J$ nondegenerate arc chains containing, respectively, 1 and 0 , then we have exactly five possibilities:

P1. $A=M=J$;

P2. $A=M$ and $\bigvee J=p$ where $0<p<1$;

P3. $A=J$ and $\wedge M=p$ where $0<\mathrm{p}<1$;

P4. $M \cap J$ is an arc chain from $p$ to $q$ with $0<p<q<1$;

P5. $M \cap J=\{p\}$ and $0<p<1$.

Difinition 3.7. Let $L$ be a compact distributive topological lattice of breadth two. Let $A_{1}$ and $A_{2}$ be arc chains from 0 to 1 satisfying for $i=1,2$ :

(1) $A_{i}=M_{i} \cup J_{i}$ 
(2) $M_{i}\left(J_{i}\right)$ is a nondegenerate arc chain containing $1(0)$ and $M(L)=M_{1}$ $\cup M_{2}\left[J(L)=J_{1} \cup J_{2}\right]$;

(3) $A_{1} \cap A_{2}=\{0,1\}$.

Then $L$ will be called an IC-lattice.

For the remainder of this paper all lattices will be assumed metrizable.

THEOREM 3.8. If $L$ is an IC-lattice and both $A_{1}$ and $A_{2}$ satisfy P5, then $L$ is iseomorphic to the direct product of two arc chains.

PRoof. Clearly $p_{1} \in M_{1} \cap J_{1}$ and $p_{2} \in M_{2} \cap J_{2}$ are complements. The theorem is thus a direct application of Lemma 2 of [5].

We shall need the following lemma.

LEMmA 3.9. Let $L$ be a bounded topological lattice with $A$ and $B$ arc chains from 0 to 1 . If

(i) $s_{B} \mid A(x)=0$ if and only if $x=0$, and

(ii) $y \in M(x)^{\circ}$ whenever $x, y \in A$ and $x<y$, then $s_{B} \mid A$ is one-to-one.

Proof. If $s_{B} \mid A$ is not one-to-one, then for some $x, y \in A$ with $0<x<y$ we have $s_{B}\left|A(x)=s_{B}\right| A(y)$. It follows easily that $\left(B \cap M(x)^{\circ}\right) \cup(B \backslash M(x))$ is a nontrivial separation of the connected set $B$.

An IC-lattice in which $A_{1}$ and $A_{2}$ both satisfy $\mathrm{P} 1$ is usually called a banana. An example in the plane is $L=\left\{(x, y): 0 \leqslant x \leqslant 1, x^{2} \leqslant y \leqslant \sqrt{x}\right\}$ with the usual topology and order of the plane.

When a chain has a subscript $C_{i}$, then we use $s_{i}$ for $s_{C_{i}}$.

THeOREM 3.10. If $L$ and $L^{\prime}$ are IC-lattices and $A_{1}, A_{2}, A_{1}^{\prime}$, and $A_{2}^{\prime}$ all satisfy $\mathrm{P} 1$, then $L$ is iseomorphic to $L^{\prime}$.

Proof. The theorem is proved by explicitly constructing an order isomorphism of $A_{1} \cup A_{2}$ onto $A_{1}^{\prime} \cup A_{2}^{\prime}$. It will then follow from Corollary 3.9 of [16] that $L$ is iseomorphic to $L^{\prime}$.

We begin the construction by partitioning the $\operatorname{arcs} A_{i}$ as follows. Let $q_{0} \in$ $A_{2} \backslash\{0,1\}$. We define

$$
\begin{aligned}
& p_{0}=t_{1}\left(q_{0}\right), \text { and for } n=1,2, \ldots, \\
& p_{2 n-1}=t_{1}\left(t_{2} t_{1}\right)^{n}\left(q_{0}\right), \\
& p_{2 n}=s_{1}\left(s_{2} s_{1}\right)^{n-1}\left(q_{0}\right), \\
& q_{2 n-1}=\left(t_{2} t_{1}\right)^{n}\left(q_{0}\right), \text { and } \\
& q_{2 n}=\left(s_{2} s_{1}\right)^{n}\left(q_{0}\right) .
\end{aligned}
$$

With these points we partition $A_{1}$ into the following subarcs:

$$
\begin{aligned}
& P(01)=A_{1} \cap\left[p_{1}, p_{0}\right], \\
& P(02)=A_{1} \cap\left[p_{0}, p_{2}\right], \text { and for } n=1,2, \ldots,
\end{aligned}
$$




$$
\begin{aligned}
& P(2 n-1,2 n+1)=A_{1} \cap\left[p_{2 n+1}, p_{2 n-1}\right], \text { and } \\
& P(2 n, 2 n+2)=A_{1} \cap\left[p_{2 n}, p_{2 n+2}\right] .
\end{aligned}
$$

We define the partition of $A_{2}$ similarly using $q_{0}, q_{1}$, etc., to obtain $Q(01)$, $Q(02)$, etc. Primes are used to denote the corresponding sets and points of $L^{\prime}$.

Let $g: P(02) \rightarrow P(02)^{\prime}$ be any iseomorphism onto $P(02)^{\prime}$. We define $f: L$ $\rightarrow L^{\prime}$ by

(i) $f \mid P(02)=g$;

(ii) $f(0)=0, f(1)=1, f\left(q_{0}\right)=q_{0}^{\prime}$;

(iii) if $x \in Q(01)$, then $f(x)=t_{2}^{\prime} g s_{1}(x)$;

(iv) if $x \in Q(02)$, then $f(x)=s_{2}^{\prime} g t_{1}(x)$;

(v) if $x \in P(01)$, then $f(x)=t_{1}^{\prime} t_{2}^{\prime} g s_{1} s_{2}(x)$.

This process may be continued inductively for each of the remaining subarcs of $A_{1}$ and $A_{2}$. By applying Lemma 3.9 above and Lemma 2.3 of [12] we see that the restrictions of the $s$ and $t$ functions to the $A_{i}$ are iseomorphisms. Thus $f$ maps a subarc of $A_{i}$ iseomorphically onto the corresponding subarc of $A_{i}^{\prime}$. Finally it is straightforward, and tedious, to verify that $f$ is an order isomorphism on $A_{1} \cup A_{2}$.

For the nonmetric case, we define $f$ on $L \backslash\left(A_{1} \cup A_{2}\right)$ by $f(x)=f\left(x_{1}\right) \wedge$ $f\left(x_{2}\right)$, where $x=x_{1} \wedge x_{2}$ is the unique representation of $x$ as a meet of meet irreducibles. Then $f$ is easily shown to be an isomorphism on $L$. To see that $f$ is continuous we observe that for each interval $[a, b] \subset L^{\prime}$,

$$
f^{-1}([a, b])=f^{-1}(M(a) \cap L(b))=M\left(f^{-1}(a)\right) \cap L\left(f^{-1}(b)\right) ;
$$

hence $f^{-1}([a, b])$ is closed. Since $L$ and $L^{\prime}$ have the interval topology [9], $f$ is continuous.

Since each of the arcs $A_{1}$ and $A_{2}$ of an IC-lattice may satisfy any one of the conditions P1 to P5, there are twenty-five lattices possible. Eighteen of these possibilities yield lattices unique in the sense of Theorem 3.10. The seven exceptions are:

(1) $A_{1}$ satisfies $\mathrm{P} 1$ and $A_{2}$ satisfies $\mathrm{P} 4$;

(2) $A_{1}$ satisfies $\mathrm{P} 2$ and $A_{2}$ satisfies $\mathrm{P} 4$;

(3) $A_{1}$ satisfies $\mathrm{P} 2$ and $A_{2}$ satisfies $\mathrm{P}$;

(4) $A_{1}$ and $A_{2}$ both satisfy P4.

The other three cases are obtained by interchanging $A_{1}$ and $A_{2}$ in (1), (2), and (3). Eliminating those cases obtained by such interchanges leaves us with fifteen distinct lattices.

Suppose $L$ is an IC-lattice with $A_{1}$ and $A_{2}$ as in case 1. Then $s_{1}(p)$ and $t_{1}(q)$ may be the same point or distinct points of $L$; see Figure 1. Thus the lattices in Figure 1 are not iseomorphic. 

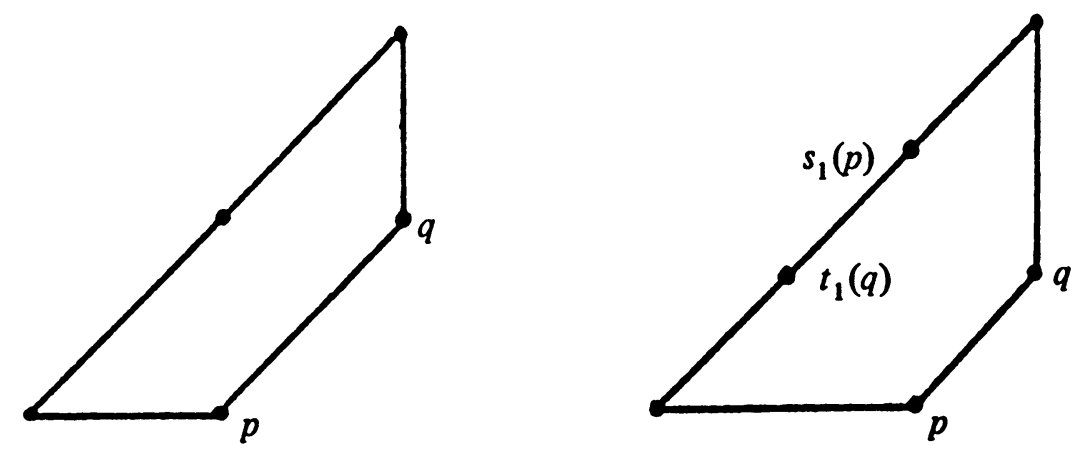

FIGURE 1

4. Nondistributive lattices. In this section we shall extend the methods of $\$ 3$ to compact connected topological lattices of breadth two for which $w(M(L))$ =3. Such lattices are modular $[6]$ but nondistributive $[2,3.1],[12,2.10]$. Examples of such lattices have been constructed in the three cell by D. E. Edmondson [7].

The difficulty in extending the methods of $\$ 3$ is the lack of uniqueness in the representation of elements as meets of two meet irreducibles. When $w(M(L))$ $=3$, the set of neutral elements of $L$ is an arc chain which can be used to recover the needed unique meet representations. An example will be given to show that if $w(M(L))>3$, then the arcs of irreducibles are not sufficient to characterize $L$.

Definition 4.1. A lattice $L$ is an $E$-lattice if $L$ is a compact connected topological lattice of breadth two with $M(L)=\bigcup_{i=1}^{3} M_{i}$ where, for $i, j=1,2,3$,

(i) $M_{i}$ is an arc chain,

(ii) $J(L)=\bigcup_{i=1}^{3} J_{i}$ and $J_{i}$ is an arc chain,

(iii) $A_{i}=M_{i} \cup J_{i}$ is an arc chain from 0 to 1 ,

(iv) $A_{i} \cap A_{j}=\{0,1\}$ when $i \neq j$,

(v) $M_{i} \cap J_{i}=z_{i} \neq 0$,

(vi) $z_{i} \wedge z_{j}=0$ when $i \neq j$.

In Definition 4.1 the chains $A_{i}$ are the boundary arcs. Thus in an $E$-lattice all the $A_{i}$ satisfy P5. As only minor modifications are needed to pass from this case to any other of the 125 possible cases, we shall present only the details of this case.

THEOREM 4.2. If $L$ is an E-lattice, then

(i) $J_{i}=\left[0, z_{i}\right] \subset M_{i} \wedge M_{j}$ when $i \neq j$, and

(ii) $p \in L$ implies $p$ belongs to at least two of $M_{1} \wedge M_{2}, M_{1} \wedge M_{3}$, and $M_{2} \wedge M_{3}$.

Proof. By Lemma 1.1 of $[17],\left[0, z_{i}\right]$ is a chain from 0 to $z_{i}$; hence $J_{i}=$ $\left[0, z_{i}\right]$ [1]. Also $z_{i} \wedge M_{j}$ is an arc chain from 0 to $z_{i}$ and so $J_{i}=z_{i} \wedge M_{j} \subset M_{i}$ $\wedge M_{j}$ when $i \neq j$. 
We note that for $i \neq j, M_{i} \wedge M_{j}$ is a distributive sublattice of $L$ [14].

Suppose $p \in M_{1} \wedge M_{2}$ and $p \notin\left(M_{1} \wedge M_{3}\right) \cup\left(M_{2} \wedge M_{3}\right)$. Letting $x_{i}=$ $s_{i}(p)$ we have $p=x_{1} \wedge x_{2} \wedge x_{3}=x_{1} \wedge x_{2} ;$ also $p<x_{1} \wedge x_{3}$ and $p<x_{2} \wedge x_{3}$. If $x_{3}=z_{3}$, then $p<x_{1} \wedge z_{3} \in\left[0, z_{3}\right] \subset M_{1} \wedge M_{3}$, contrary to the assumption that $p \notin M_{1} \wedge M_{3}$. If $x_{3} \neq z_{3}$, then there exists an $x \in M_{3}$ such that $x<x_{3}$; then $x_{1} \wedge x_{2} \wedge x<p$. Since $L$ has breadth two, $x_{1} \wedge x_{2} \wedge x=x_{1} \wedge x$ or $x_{2}$ $\wedge x$. Thus, since $M_{3}$ is order dense, there exists a net $\left\{x_{\alpha}\right\}_{\alpha \in \Delta}$ converging to $x_{3}$ such that $x_{\alpha}<x_{3}$ and $x_{1} \wedge x_{2} \wedge x_{\alpha}=x_{1} \wedge x_{\alpha}$ or $x_{2} \wedge x_{\alpha}$.

Thus, for some cofinal subset $\Gamma \subset \Delta, x_{1} \wedge x_{2} \wedge x_{\alpha}=x_{1} \wedge x_{\alpha}$ for all $\alpha \in$ $\Gamma$ or $x_{1} \wedge x_{2} \wedge x_{\alpha}=x_{2} \wedge x_{\alpha}$ for all $\alpha \in \Gamma$. If $x_{1} \wedge x_{2} \wedge x_{\alpha}=x_{1} \wedge x_{\alpha}$ for all $\alpha \in \Gamma$, then the net $\left\{\left(x_{1} \wedge x_{\alpha}, p\right)\right\}_{\alpha \in \Gamma}$ converges to $\left(x_{1} \wedge x_{3}, p\right)$. Thus $x_{1} \wedge$ $x_{3} \leqslant p<x_{1} \wedge x_{3}$. We conclude that $p \in\left(M_{1} \wedge M_{3}\right) \cup\left(M_{2} \wedge M_{3}\right)$.

THEOREM 4.4. If $L$ is an E-lattice and if $A=\bigcap_{i \neq j}\left(M_{i} \wedge M_{j}\right)$, then (i) $A$ is an arc chain from 0 to 1 , and (ii) $x, y \in A$ and $x<y$ imply $y \in M(x)^{\circ}$.

Proof. Clearly $A$ is a compact chain. By Theorem 4.2, $L=\left(M_{1} \wedge M_{2}\right)$ $\cup\left(M_{2} \wedge M_{3}\right)$. Since $L$ is unicoherent, then $\left(M_{1} \wedge M_{2}\right) \cap\left(M_{2} \wedge M_{3}\right)$ is connected. Again from Theorem 4.2, $L=\left(M_{1} \wedge M_{3}\right) \cup\left[\left(M_{1} \wedge M_{2}\right) \cap\left(M_{2} \wedge M_{3}\right)\right]$; thus $A$ is connected.

If $x, y \in A$ and $x<y$, then $s_{i}(x)<s_{i}(y)$. By Theorem 3.8, $M_{i} \wedge M_{j}$ is iseomorphic to $M_{i} \times M_{j}$. The image of $y,\left(s_{i}(y), s_{j}(y)\right)$, is in the interior of $M\left(\left(s_{i}(x), s_{j}(x)\right)\right)$. Since $L=\bigcup\left(M_{i} \wedge M_{j}\right)(i \neq j)$, it follows that $y \in M(x)^{\circ}$.

The function $t_{A} \mid M_{i}$ is an iseomorphism with inverse $s_{i} \mid A$ for $i=1,2,3$. For convenience we drop the subscript $A$. Note that for each $x \in L$, both $x$ and $t\left(s_{i}(x)\right)$ belong to the same chain $M_{j} \wedge\left(s_{i}(x)\right)$ for some $j \neq i$. Thus $x$ and $t\left(s_{i}(x)\right)$ are related.

THEOREM 4.5. Let $L$ be an E-lattice. If $x=x_{1} \wedge x_{3}=x_{2} \wedge x_{3} \neq x_{1} \wedge x_{2}$, where $x_{i}=s_{i}(x)$, then $x=t\left(x_{1}\right) \wedge x_{i}$ and $t\left(x_{1}\right)=t\left(x_{2}\right)$.

Proof. If $t\left(x_{1}\right) \leqslant x$, then $x \in\left[t\left(x_{1}\right), x_{1}\right] \subset M_{1} \wedge M_{2}$ contrary to $x \neq x_{1}$ $\wedge x_{2}$. Hence $x<t\left(x_{1}\right)$, and so $x=x_{1} \wedge x_{3} \leqslant t\left(x_{1}\right) \wedge x_{3} \leqslant x_{1} \wedge x_{3}=x$. Similarly $x=t\left(x_{2}\right) \wedge x_{3}$. Since $s_{1}$ is an isomorphism on $M_{1} \wedge M_{3}$ and $s_{i}\left(x_{j}\right)=1$, we have $s_{1}\left(t\left(x_{1}\right)\right)=s_{1}\left(t\left(x_{1}\right)\right) \wedge s_{1}\left(x_{3}\right)=s_{1}\left(t\left(x_{1}\right) \wedge x_{3}\right)=s_{1}\left(t\left(x_{2}\right) \wedge x_{3}\right)=$ $s_{1}\left(t\left(x_{2}\right)\right)$. Thus $t\left(x_{1}\right)=t\left(x_{2}\right)$.

TheOREM 4.6. If $L$ and $L^{\prime}$ are two E-lattices, then $L$ is iseomorphic to $L^{\prime}$.

Proof. Let $A, M_{i}, J_{i}, t$, and $s_{i}$ be the sets and functions of the preceding discussion for $L$ with primes denoting the corresponding sets and functions for $L^{\prime}$. Let $g: A \rightarrow A^{\prime}$ be any iseomorphism onto $A^{\prime}$. We extend $g$ to $f: L \rightarrow L^{\prime}$ by 
(i) $f \mid A=g$,

(ii) if $x \in M_{i}$, then $f(x)=s_{i}^{\prime} g t(x)$, and

(iii) if $x \in L \backslash\left[\left(\cup_{i=1}^{3} M_{i}\right) \cup A\right]$, then $f(x)=g t\left(x_{i}\right) \wedge s_{i}^{\prime} g t\left(x_{j}\right)$, where $x=$ $t\left(x_{i}\right) \wedge x_{j}=x_{i} \wedge x_{j}=s_{i}(x) \wedge s_{j}(x)$.

From Theorem 4.5 we see that $f$ is independent of the representation $t\left(x_{i}\right)$ $\wedge x_{j}$ chosen for $x$. The proof that $f$ is an iseomorphism on each $M_{i} \wedge M_{j}$ is similar to the proof of Theorem 3.10. If $x, y \in L$, then $x, y \in M_{i} \wedge M_{j}$ for some $i \neq j$. Since $f$ is an isomorphism on $M_{i} \wedge M_{j}$, then $f(x \vee y)=f(x) \vee f(y)$ and $f(x \wedge y)=f(x) \wedge f(y)$.

Example 4.7. Let $T_{1}$ and $T_{2}$ be two copies of the unit square $I_{2}$. We let $L$ be the topological space obtained by identifying corresponding points of the region on and between the arcs $y=x^{2}$ and $y=\sqrt{x}$. Thus $L$ consists of five regions which we identify as follows. Let $A_{i}=\left\{(x, y) \in T_{i}: 0 \leqslant x \leqslant 1, \sqrt{x} \leqslant\right.$ $y \leqslant 1\}$ and $B_{i}=\left\{(x, y) \in T_{i}: 0 \leqslant x \leqslant 1,0 \leqslant y \leqslant x^{2}\right\}$. The fifth region is $T_{1}$ $\cap T_{2}=\left\{(x, y): 0 \leqslant x \leqslant 1, x^{2} \leqslant y \leqslant \sqrt{x}\right\}$. In $\S 3$ we showed that $A_{i}$ and $B_{i}$ are each iseomorphic to $P=\left\{(x, y) \in I_{2}: 0 \leqslant x \leqslant 1, x \leqslant y \leqslant 1\right\}$ and $Q=$ $\left\{(x, y) \in I_{2}: 0 \leqslant x \leqslant 1,0 \leqslant y \leqslant x\right\}$. Thus we may consider $A_{1} \cup A_{2}$ to be a copy of $I_{2}$ with $A_{1}=P$ and $A_{2}=Q$. Similarly for $B_{1} \cup B_{2}$ with $B_{1}=Q$ and $B_{2}=P$.

We now describe a meet operation for $L$; joins are defined similarly. Let $a, b \in L$.

Case 1. If $a, b \in T_{i}$, then $a \wedge b$ is the meet of $a$ and $b$ in $T_{i}$.

Case 2. If $a \in A_{1}$ and $b \in A_{2}$, then $a$ and $b$ are mapped to $P \cup Q$ and the meet of their images is mapped back to $A_{1} \cup A_{2}$ to provide a meet for $a$ and $b$. The procedure for $a \in B_{1}$ and $b \in B_{2}$ is similar.

Case 3. If $a \in A_{1}$ and $b \in B_{2}$, then we let $b^{\prime}$ be that point of $B_{1}$ which corresponds to $b$; e.g. $(1 / 2,1 / 4)$ may be considered a point of $B_{1}$ or $B_{2}$. We compute $c=a \wedge b^{\prime}$ in $T_{1}$. If $c \notin B_{1}$, then $c$ is the meet of $a$ and $b$ in $L$. If $c \in B_{1}$, then the corresponding point of $B_{2}$ is the meet of $a$ and $b$ in $L$. The case of $a \in$ $A_{2}$ and $b \in B_{1}$ is handled similarly.

If $M_{i}=\left\{(x, 1) \in T_{i}: 0 \leqslant x \leqslant 1\right\}$ and $M_{i}^{\prime}=\left\{(1, y) \in T_{i}: 0 \leqslant y \leqslant 1\right\}$, then the $M_{i}$ and $M_{i}^{\prime}$ are the arc chains of meet irreducibles of $L$. Also $\left(M_{1} \wedge M_{2}\right) \cap$ $\left(M_{1}^{\prime} \wedge M_{2}^{\prime}\right)=\{0,1\}$.

In Edmondson's example constructed from four copies of the unit interval, $\bigcap_{i \neq j}\left(M_{i} \wedge M_{j}\right)$ is an arc chain from 0 to 1 [7]. Thus the nice characterization for lattices $L$ with $w(M(L)) \leqslant 3$ does not generalize directly to lattices $L$ with $w(M(L))>3$.

Portions of this paper appeared in [11].

\section{REFERENCES}

1. L. W. Anderson, On the distributivity and simple connectivity of plane topological lattices, Trans. Amer. Math. Soc. 91 (1959), 102-112. MR 21 \#1365. 
2. K. A. Baker and A. R. Stralka, Compact, distributive lattices of finite breadth, Pacific J. Math. 34 (1970), 311-320. MR 44 \#129.

3. G. Birkhoff, Lattice theory, 3rd ed., Amer. Math. Soc. Colloq. Publ., vol. 25, Amer. Math. Soc., Providence, R. I., 1967. MR 37 \#2638.

4. D. R. Brown, Topological semilattices on the two-cell, Pacific J. Math. 15 (1965), 35-46. MR 31 \#725.

5. T. H. Choe, Locally compact lattices with small lattices, Michigan Math. J. 18 (1971), $81-85$.

6. D. E. Edmondson, Modularity in topological lattices, Proc. Amer. Math. Soc. 21 (1969), 81-82. MR 39 \#2132. \#4062.

7. - A modular topological lattice, Pacific J. Math. 29 (1969), 271-277. MR 39

8. A. Y. W. Lau, Remarks on semilattices on the two-cell (preprint).

9. J. D. Lawson, Intrinsic topologies in topological lattices and semilattices, Pacific J. Math. 44 (1973), 593-602. MR 47 \#6580.

10. J. D. Lawson and B. Madison, Peripheral and inner points, Fund. Math. 69 (1970), 253-266. MR 43 \#1175.

11. J. W. Lea, Jr., Irreducible elements in compact topological lattices, Dissertation, Louisiana State Univ., Baton Rouge, Louisiana, 1971.

12. - An embedding theorem for compact semilattices, Proc. Amer. Math. Soc. 34 (1972), 325-331.

13. - The peripherality of irreducible elements of a lattice, Pacific J. Math. 45 (1973), 555-560. MR 47 \#4875.

14. - Sublattices generated by chains in modular topological lattices, Duke Math. J. 41 (1974), $241-246$.

15. K. Oberhoff, Semilattice structures on certain non-metric continua, Dissertation, Univ. of Houston, Houston, Texas, 1973.

16. J. B. Rhodes, Decomposition of semilattices with applications to topological lattices, Pacific J. Math. 44 (1973), 299-307. MR 47 \#3262.

17. E. D. Shirley and A. R. Stralka, Homomorphisms on connected topological lattices, Duke Math. J. 38 (1971), 483-490. MR 43 \#5497.

18. A. D. Wallace, Factoring a lattice, Proc. Amer. Math. Soc. 9 (1958), 250-252. MR $20 \# 825$.

DEPARTMENT OF MATHEMATICS, MIDDLE TENNESSEE STATE UNIVERSITY, MURFREESBORO, TENNESSEE 37132 